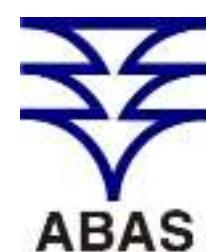

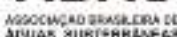
mww.abas.org

\section{ELETRORRESISTIVIDADE NA PROSPECÇÃO DE AQUÍFEROS FRATURADOS}

\author{
PROSPECTING FRACTURED AQUIFERS WITH ELECTRICAL \\ RESISTIVITY
}

\author{
José Domingos Faraco Gallas ${ }^{1}$; Arnoldo Giardin ${ }^{2}$
}

Artigo recebido em: 12/08/2015 e aceito para publicação em: 03/05/2016.

DOI: http://dx.doi.org/10.14295/ras.v30i1.28496

\begin{abstract}
Resumo: Levantamentos geofísicos de eletrorresistividade com arranjo dipolo-dipolo em áreas de ocorrência de rochas granito-gnáissicas foram realizados visando a detecção de estruturas do tipo falhas/fraturas ou outras de interesse hidrogeológico. Os resultados obtidos permitiram identificar estruturas que, em perfurações subsequentes, resultaram em 2 poços com excelente produção de água. Um terceiro poço foi perfurado, mas em local onde a assinatura geofísica poderia refletir um depósito de tálus, potencial bom aquífero ou rochas xistosas, potencial mau aquífero, sendo confirmada esta segunda possibilidade.
\end{abstract}

Palavras-chave: Resistividade. Água subterrânea. Aquíferos fraturados.

\begin{abstract}
Geophysical resistivity surveys in granite-gneiss rocks, with dipole-dipole array, were carried out to detect fault/fracture structures or other of hydrogeological interest. The geophysical data allowed the identification of structures that in subsequent drilling resulted in two wells with excellent production volumes of water. A third well was drilled at a local where the geophysical data revealed an ambiguous geophysical signature which could reflect a talus, potentially a good aquifer, or a schist rock, potentially a bad aquifer, which was indeed confirmed.
\end{abstract}

Keywords: Resistivity. Groundwater. Fractured aquifers.

\section{INTRODUÇÃO}

A água é o mais precioso bem mineral e sem ela não há vida. Nos dias atuais, de crises hídricas, de aumento da população e da poluição dos mananciais superficiais, além de sua potencial exaurição, tem-se intensificado a prospecção de aquíferos subterrâneos, tanto em áreas sedimentares como de embasamento cristalino, aquíferos porosos e aquíferos fraturados, respectivamente.

O presente levantamento geofísico foi efetuado em área de rochas granitognáissicas de embasamento cristalino, visando principalmente a prospecção de zonas de fraturadas, potenciais aquíferos.

Para tanto, foi empregado o método da eletrorresistividade com o dispositivo eletródico dipolo-dipolo.

Os dados de descrição das litologias interceptadas pelas perfurações confirmaram a presença de granitos, gnaisses, mafitos, felsitos e brechas, conforme visto nas figuras 12,13 e 14 .

A presença de zonas fraturadas nas rochas subjacentes é indicada pela detecção de resistividades mais baixas. Estas resistividades mais baixas devem-se ao fato que uma rocha fraturada/alterada con-

1 Geólogo pela UFRGS em 1978, mestre em Geofísica pela UFPa em 1990 e Doutor pela UNESP em 2000. Pesquisador III até 2002 no IPT e Professor Doutor do Departamento de Gelogia Sedimentar e Ambiental do IGc USP desde 2002 (jgallas@usp.br)

2 Geólogo pela UFRGS formado em 1982, trabalhou na CORSAN (Companhia Riograndense de Saneamento) até 2012. Atualmente atua na empresa Natureza Líquida Prestação de Serviços em Geologia Ltda (n1001@naturezaliquida.com.br) 
tém um eletrólito constituído de água com íons dissolvidos e/ou material particulado em suspensão, que irão ocasionar quedas nas resistividades, principalmente quando compradas às da rocha sã encaixante.

Nos locais de baixas resistividades detectados foram efetuadas perfurações que confirmaram a presença de excepcionais aquíferos e foram instalados poços para explotação das águas com excelente qualidade - classificadas como minerais e produtividades bastante elevadas.

\section{OBJETIVOS}

Os levantamentos geofísicos de eletrorresistividade objetivaram detectar anomalias ou assinaturas geofísicas correlacionáveis a falhas/fraturamentos ou outras estruturas favoráveis à locação de poços tubulares para captação de águas subterrâneas.

\section{GEOLOGIA E CONSIDERAÇÕES HIDROGEOLÓGICAS}

A área onde foram realizados os trabalhos situa-se no município de Duque de Caxias, Rio de Janeiro.

Conforme reconhecimentos de campo e consulta ao mapa geológico de detalhe 1:25.000 (Figura 4) e do mapa 1:500.000 do Estado do Rio de Janeiro, ocorrem na área somente rochas de embasamento cristalino. São granitos/granodioritos neoproterozóicos, sienitos, foiaitos cambrianos e gnaisses meso/neoproterozóicos.

As figuras 12, 13 e 14, que contêm as descrições litológicas que as sondagens interceptaram, confirmam a presença destes tipos rochosos.

Neste contexto litológico os aquíferos somente podem ocorrer em zonas fraturadas, uma vez que estas rochas, quando não fraturadas, têm porosidades próximas de zero. Outra possibilidade de ocorrência de aquíferos nestas condições seria em depósitos aluvionares ou de tálus, conforme comentado nos itens 6 e 9 .

\section{MÉTODO E TÉCNICAS UTILIZA- DOS}

\subsection{Eletrorresistividade}

De acordo com a segunda lei de Ohm, a resistência elétrica $\boldsymbol{R}$ de um condutor homogêneo é proporcional ao seu comprimento $\boldsymbol{l}$ e inversamente proporcional à área de sua seção transversal $\boldsymbol{A}$.

$$
R=\frac{\rho l}{A}
$$

onde $\boldsymbol{\rho}$ é a resistividade elétrica, expressa em ohms x metro.

\subsection{Resistividade Aparente ( $\rho a)$}

Trata-se de um dos conceitos fundamentais em prospecção geoelétrica. Em um levantamento de campo de eletrorresistividade são medidos a corrente $\boldsymbol{I}$, injetada ao subsolo por meio de um par de eletrodos $\boldsymbol{A}$ e $\boldsymbol{B}$, e a diferença de potencial $\Delta \boldsymbol{V}$, mensurada por um segundo par de eletrodos $\boldsymbol{M}$ e $\boldsymbol{N}$. De posse destes parâmetros é obtida a resistividade $\rho$. Considerando um terreno homogêneo e isotrópico, a resistividade será constante independentemente dos posicionamentos dos eletrodos empregados nas medições. A Figura 1 ilustra sinteticamente o método da eletrorresistividade.

No entanto, em condições naturais, os substratos não têm comportamentos homogêneos e isotrópicos. No caso dos dois pares de eletrodos (emissão de $\boldsymbol{I}$ e recepção de $\boldsymbol{\Delta} \boldsymbol{V}$ ) estarem em locais de resistividades $\rho_{1}, \rho_{2}, \rho_{3}$ e $\rho_{4}$, (figura 2), a resistividade final medida não será a verdadeira e sim uma resistividade chamada aparente $\rho_{a}$ (Orellana, 1972). Esta resistividade não será igual a nenhuma das quatro, mas será uma contribuição de todas e de suas geometrias. 


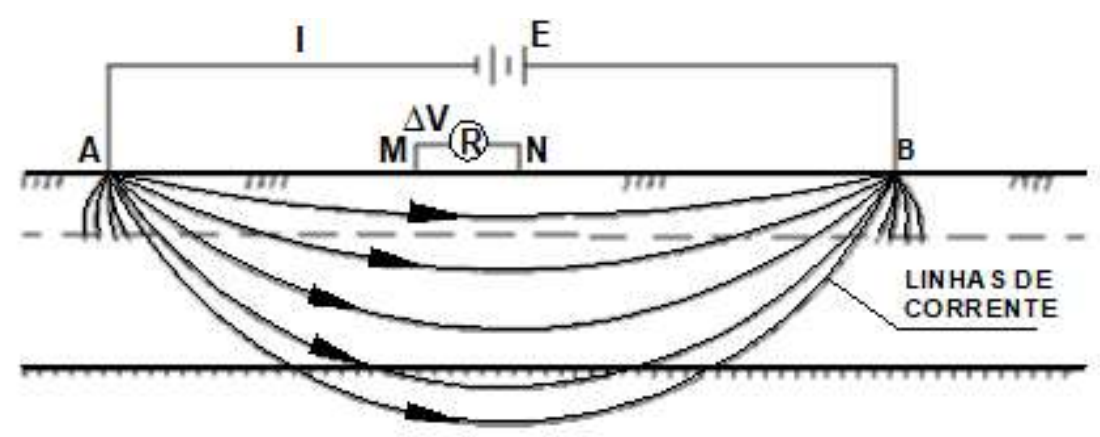

Figura 1 - Princípio do método da eletrorresistividade Figure 1 - Principle of the resistivity method

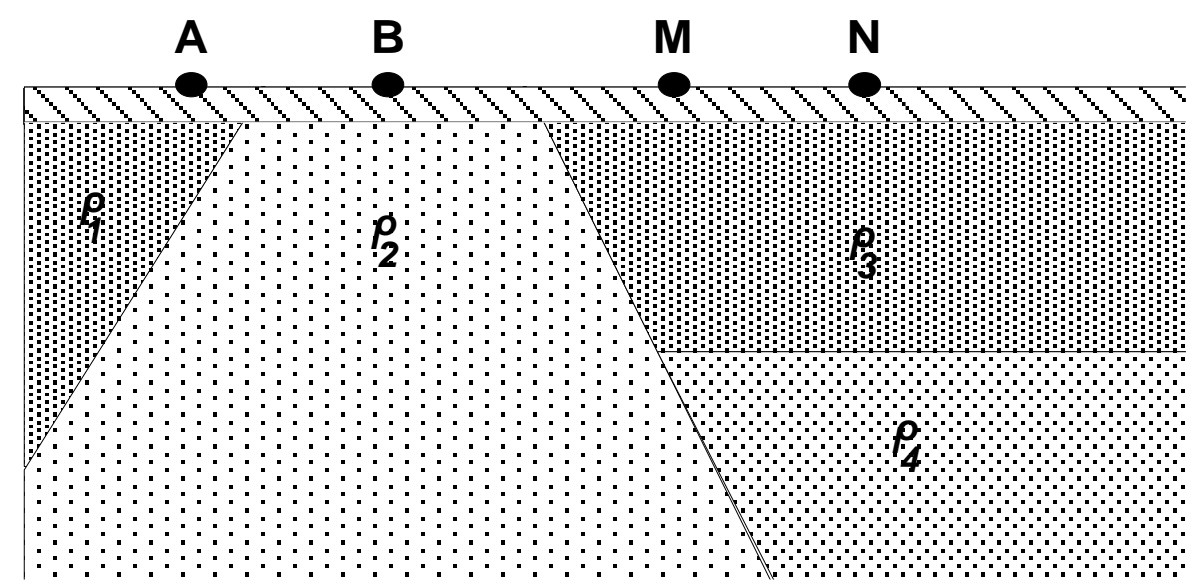

Figura 2 - Substrato heterogêneo (Gallas, 2000, modificado de Orellana, 1972)

Figure 2 - Heterogeneous substrate (Gallas, 2000 modified by Orellana, 1972)

Esta resistividade também não será a média nem a média ponderada das $\rho_{\boldsymbol{l}}$, $\rho_{2}, \rho_{3}$ e $\rho_{4}$, podendo acontecer que seja maior ou menor que qualquer uma delas (Orellana, op. cit.)

A resistividade aparente resultante de medidas realizadas sobre um meio heterogêneo é obtida empregando-se a relação usada para meios homogêneos. Esta equação considera a injeção de corrente por meio dos eletrodos A e $\mathbf{B}$ e dois pontos de medidas de potencial ( $\mathbf{M}$ e $\mathbf{N}$ ). Estabelece-se que um dos pontos seja negativo (B, p. ex.) e o outro positivo (A, p. ex.).

Sendo o potencial em B negativo, supõe-se que a corrente entra em A e sai em B.

$$
\rho=\left(\frac{U_{M}-U_{N}}{I}\right) \frac{2 \pi}{\frac{1}{A M}-\frac{1}{B M}-\frac{1}{A N}+\frac{1}{B N}}=K \frac{\Delta U}{I}
$$

Desta forma, os valores de $\rho$ calculados são os valores de $\rho_{a}$ e são calculados em função da separação entre eletrodos por meio do fator geométrico $\boldsymbol{K}$, expresso como

$$
K=\frac{2 \pi}{\frac{1}{A M}-\frac{1}{B M}-\frac{1}{A N}+\frac{1}{B N}}
$$

Finalmente, pode-se então expressar a resistividade aparente como sendo

$$
\rho_{a}=K \frac{\Delta U}{I}
$$

\subsection{Arranjo dipolo-dipolo/pseudo-seções de resistividade}

As pseudo-seções são assim chamadas porque os dados obtidos de diferentes níveis de investigação não correspondem aos valores reais dos parâmetros de cada camada investigada e referem-se a valores aparentes 
de resistividade. De forma semelhante, as profundidades na seção vertical são também apenas qualitativas.

$\mathrm{Na}$ interpretação dos dados das pseudo-seções obtêm-se informações qualitativas sobre a posição espacial dos corpos em subsuperfície e, mais raramente, pode-se estimar o seu mergulho. Como decorrência, as informações são tão mais precisas quanto melhor a definição de uma anomalia de resistividade.

O arranjo eletródico de pseudoseções utilizado neste trabalho foi o dipolo-dipolo. Neste arranjo, os eletrodos A e $\mathrm{B}$ de envio de corrente e os eletrodos $\mathrm{M}$ e $\mathrm{N}$ de potencial ou de recepção, são alinhados sobre um mesmo perfil. $\mathrm{O}$ arranjo é definido pelos espaçamentos $1=\mathrm{AB}=$ MN. A profundidade de investigação cresce com $(n+1) l / 2$ (Figura 3$)$.

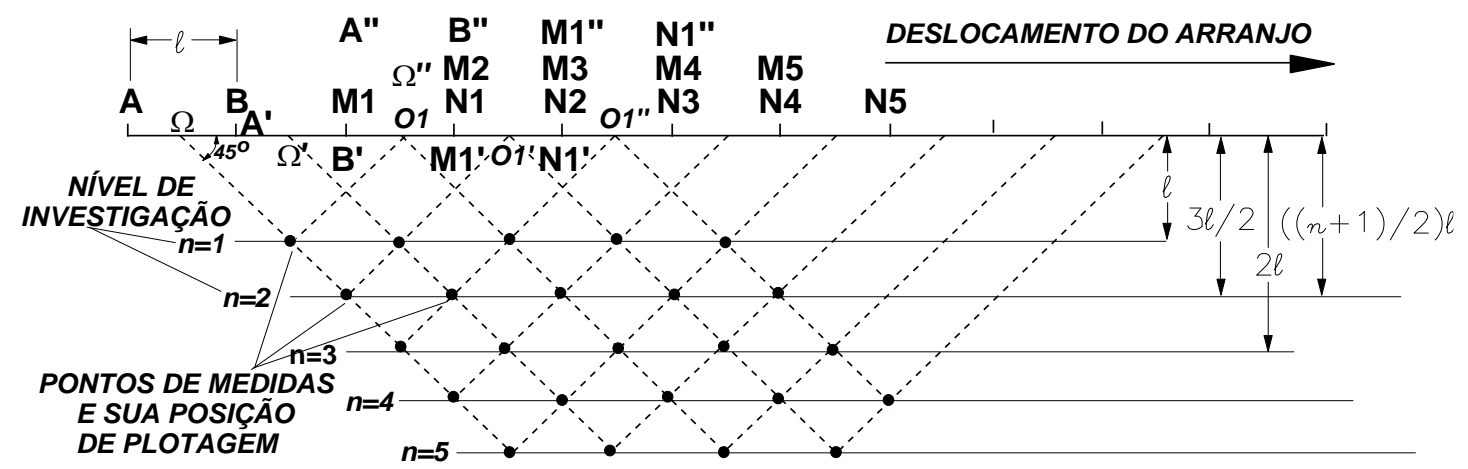

Figura 3 - Caminhamento Resistividade/Disposição do arranjo dipolo-dipolo (Gallas, 2000, adaptado de Hallof, 1957)

Figure 3 - Profiling Resistivity / layout of dipole-dipole (Gallas 2000, adapted from Hallof, 1957)

Os pontos de plotagem são atribuídos na intersecção a $45^{\circ}$ das origens $\boldsymbol{\Omega} \mathrm{e} \boldsymbol{O}$ de cada dipolo, indicando a profundidade teórica atingida por aquele ponto de medida (profundidade $=(n+1) l / 2$, sendo $n=1$; 2; 3 etc). Para que isto seja executado, realiza-se o seguinte procedimento: é mantida uma posição fixa dos eletrodos de emissão de corrente $\boldsymbol{A} \boldsymbol{B}$ e são efetuadas uma série de medidas movendo-se os eletrodos de potencial $\boldsymbol{M N}$ ao longo do perfil de medidas com deslocamentos iguais a $\boldsymbol{l}$ : $M_{1} N_{1}(n=1) ; M_{2} N_{2}(n=2) ; M_{3} N_{3}(n=3)$ e assim sucessivamente.

A cada estação, os dois dipolos são deslocados a uma distância igual a 1, e os dados obtidos são plotados nas posições $n$ $=1,2,3, \ldots$ e interpolados, gerando uma pseudo-seção de resistividade aparente.

Atualmente, os resultados das pseudo-seções são processados em programas de inversão de dados em que são obtidas seções modeladas de resistividade que, em tese, reproduzem de uma maneira realista, a distribuição das resistividades em subsuperfície, mais facilmente correlacionáveis à geologia do local estudado.

O software comumente empregado para a inversão dos dados das pseudoseções é o RES2DINV(2001), da Geotomo Software, versão 3.4, 2D Resistivity and IP Inversion e que foi o empregado neste trabalho.

\section{DIMENSIONAMENTO E EXECU- ÇÃO DOS ENSAIOS GEOFÍSI- COS}

A disposição dos perfis/malha dos levantamentos ideal é estabelecê-los conforme uma interpretação preliminar de levantamentos aerofotogramétricos, se disponíveis, de forma a posicionar os levantamentos ortogonalmente às estruturas previamente interpretadas. Mapas geológicos e/ou topográficos também podem ser úteis para este objetivo.

Informações de ordem geológica/estruturais podem ser de excepcional utilidade. Por vezes são de fundamental 
importância no sentido de conhecer-se as direções preferenciais sobre direções de foliação e direções dos movimentos relativos que causaram as fraturas (fraturas "fechadas" ou "abertas").

Se não houver nenhuma informação deste tipo, resta a solução de posicionar a malha/perfis de forma a interceptar as estruturas quaisquer que sejam as suas direções. A disposição dos perfis pode ser triangular ou quadrangular, o que irá assegurar que as estruturas sejam detectadas independentemente das orientações das mesmas.

Felizmente, no presente trabalho, havia um conhecimento geológico-estrutural da área de interesse de forma que foi possível estabelecer a malha em uma direção que interceptou praticamente de forma perpendicular as estruturas.

A Figura 4 (mapa geológico 1:25.000) apresenta sinteticamente a geologia local e também as direções das estruturas, que são NE-SW. O mapa geológico 1:500.000 do Estado do Rio de Janeiro mostra que na região estudada há uma outra direção de estruturas, praticamente perpendicular, aproximadamente SE-NW.

Desta forma, os posicionamentos praticamente E-W dos perfis geofísicos irão certamente interceptar as eventuais estruturas NE e SE identificadas preliminarmente conforme as informações geológicas dos vista nos mapa citados.

Também deve-se dar especial atenção ao dimensionamento do arranjo de eletrodos que vai ser empregado, dipolo-dipolo, neste caso.
Considerando a profundidade teórica proposta por Hallof (1957), o espaçamento entre os eletrodos deve ser de forma que já no primeiro ou no máximo no segundo nível de investigação seja ultrapassado o pacote superficial constituído por solo+rocha alterada (Gallas, 2003).

Assim, conforme este critério, os ensaios de eletrorresistividade foram executados com abertura entre eletrodos de $30 \mathrm{~m}(\mathrm{AB}=\mathrm{MN}=30 \mathrm{~m})$ e 6 profundidades teóricas de investigação $(\mathrm{n} 1=30 \mathrm{~m} ; \mathrm{n} 2=45$ $\mathrm{m} ; \mathrm{n} 3=60 \mathrm{~m} ; \mathrm{n} 4=75 \mathrm{~m}, \mathrm{n} 5=90 \mathrm{~m}$ e $\mathrm{n} 6=105$ m (Hallof, 1957).

As extensões lineares dos trabalhos foram:

Linha A: estaca 0 até estaca 2250; $2.550 \mathrm{~m}$.

Linha B: estaca 0 até estaca 2430; $2.430 \mathrm{~m}$.

Linha C: estaca 0 até estaca 570 ; $570 \mathrm{~m}$.

Total Final: 5.550m lineares de levantamento dipolo-dipolo.

\section{APRESENTAÇÃO DOS RESULTA- DOS}

Os resultados obtidos no trabalho realizado estão contidos nas figuras listadas a seguir. As figuras que contêm as linhas A e B mostram os dois trechos mais relevantes dos resultados obtidos, uma vez que os perfis ensaiados são muito extensos e, se apresentados em sua totalidade, dificultariam uma visão adequada dos resultados mais significativos aos objetivos do trabalho. 


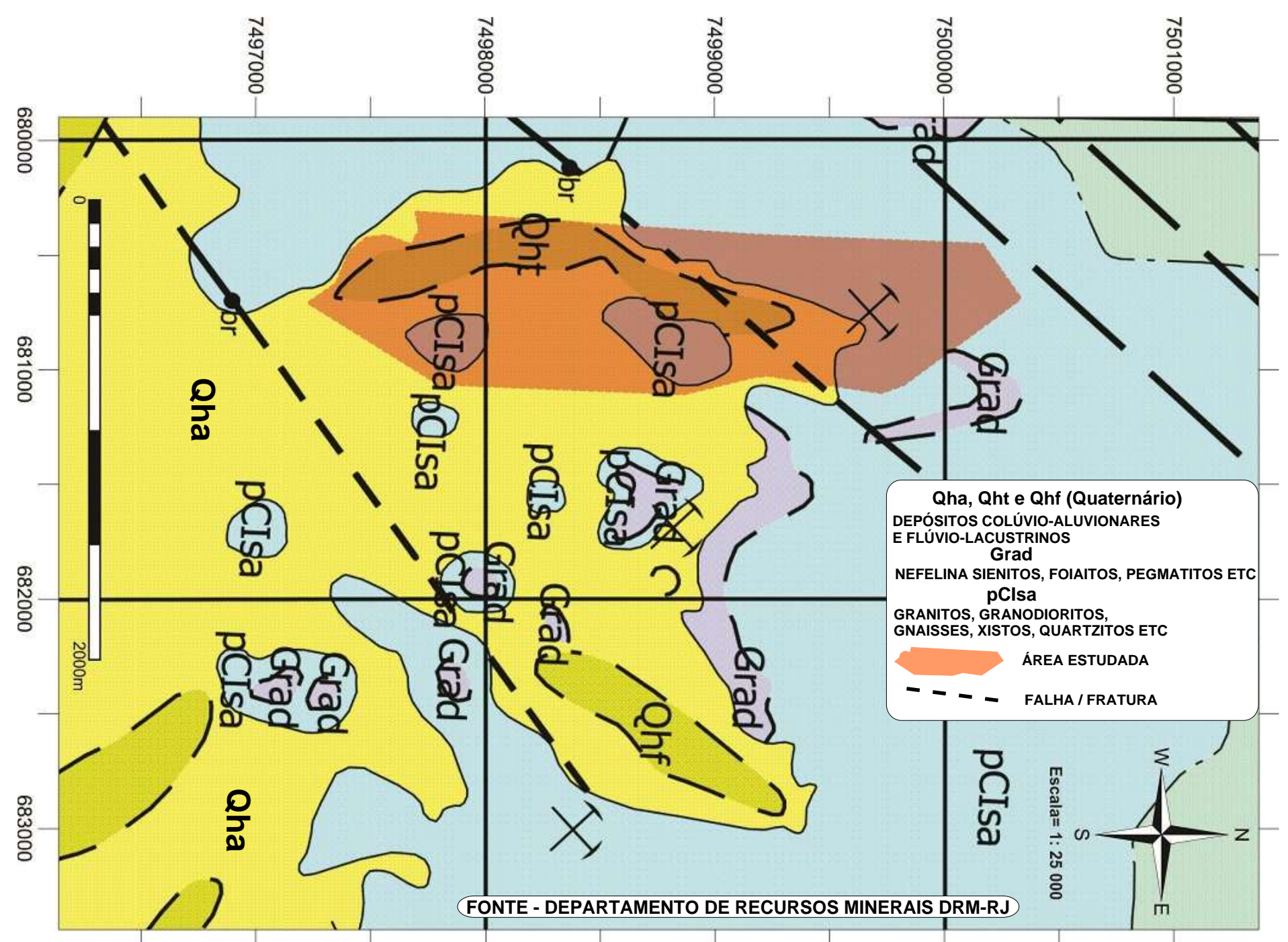

Figura 4 - Mapa geológico da área

Figure 4 - Geological map of the area 


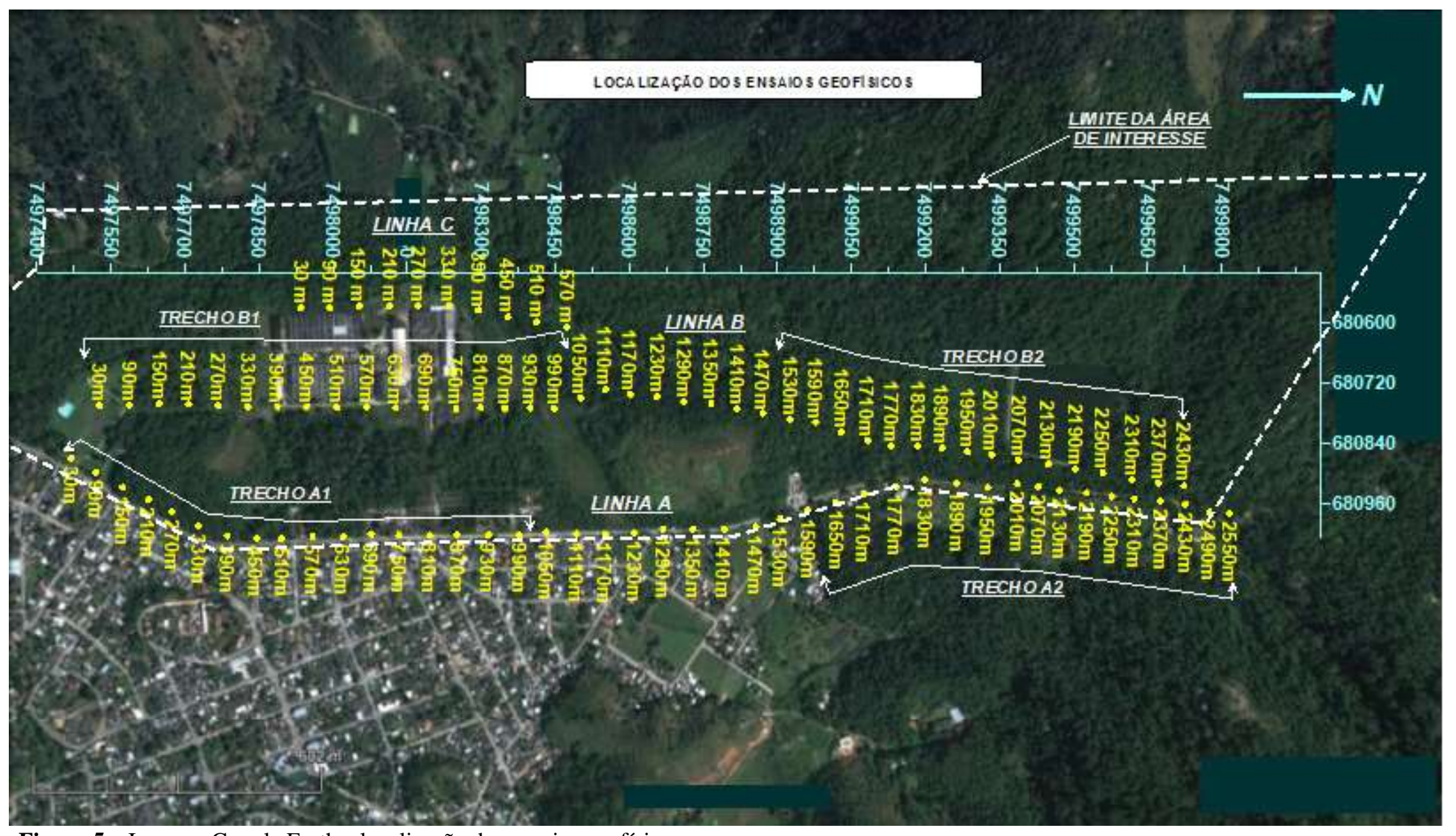

Figura 5 - Imagem Google Earth e localização dos ensaios geofísicos

Figure 5 - Google Earth Image and geophysical surveys location 


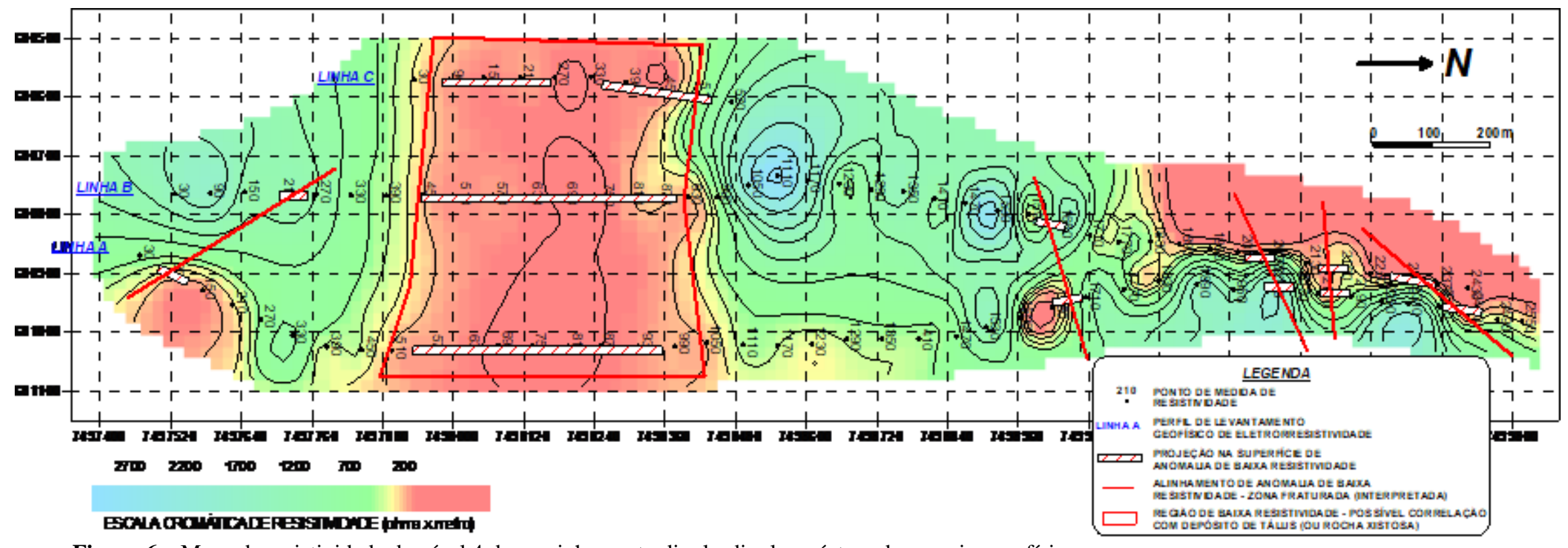

Figura 6 - Mapa de resistividade do nível 4 do caminhamento dipolo-dipolo e síntese dos ensaios geofísicos

Figure 6 - Level 4 resistivity map of dipole-dipole profiling and synthesis of geophysical surveys 
LEVANTAMENTO DE ELETRORRESISTIVIDADE, ARRANJO DIPOLO-DIPOLO - LINHA A - AB $=M N=30 \mathrm{~m}$
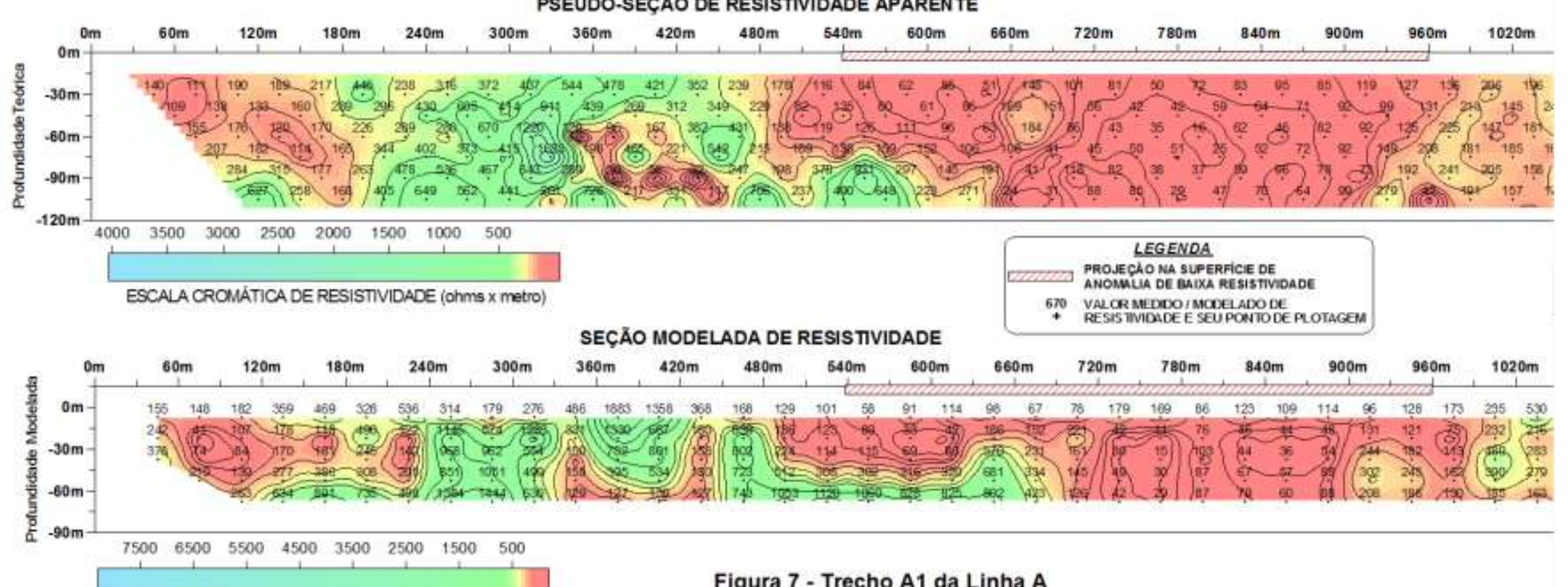

Figura 7 - Trecho A1 da Linha A

ESCALA CROMÁTICA DE RESISTIVDADE (ohms $x$ metro)

Figura 7 - Trecho A1 da pseudo-seção e seção modelada de resistividade da Linha A

Figure 7 - Part A1 of pseudo-section and modeled section of resistivity of Line A 
LEVANTAMENTO DE ELETRORRESISTIVIDADE, ARRANJO DIPOLO-DIPOLO - LINHA A - $A B=M N=30 \mathrm{~m}$

PSEUDO-SEÇÃO DE RESISTIVIDADE APARENTE
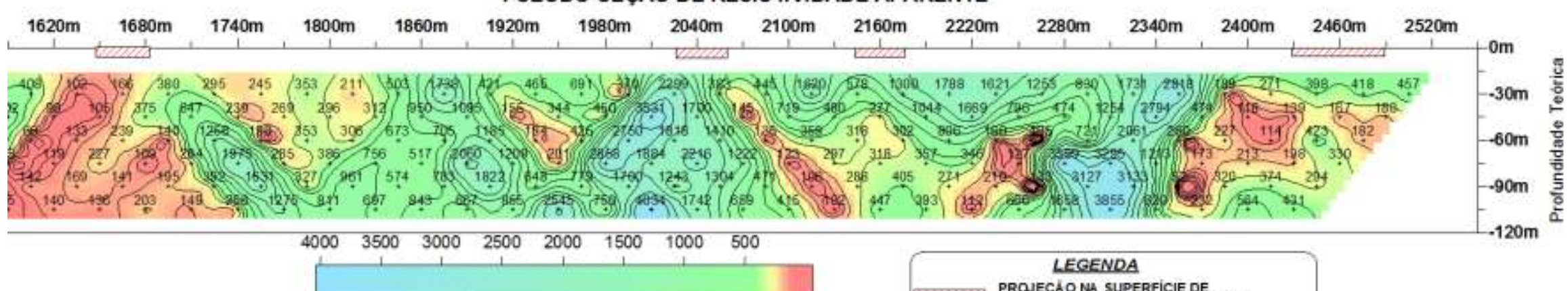

ESCALA CROMÁTICA DE RESISTIVIDADE (ohms $\times$ metro)

\section{LEGENDA}

SEÇÄO MODELADA DE RESISTIVIDADE

\begin{tabular}{|c|c|}
\hline & $\begin{array}{l}\text { LEGENDA } \\
\text { PROJECAONM SUPERFICIE DE } \\
\text { ANOMALIA DE BAIXA RESISTIVIDADEE }\end{array}$ \\
\hline$\stackrel{670}{*}$ & $\begin{array}{l}\text { VALOR MEDIDO/MODELADO DE } \\
\text { RESISTIVIDADE E SEU PONTO DE PLOTAGEM }\end{array}$ \\
\hline
\end{tabular}

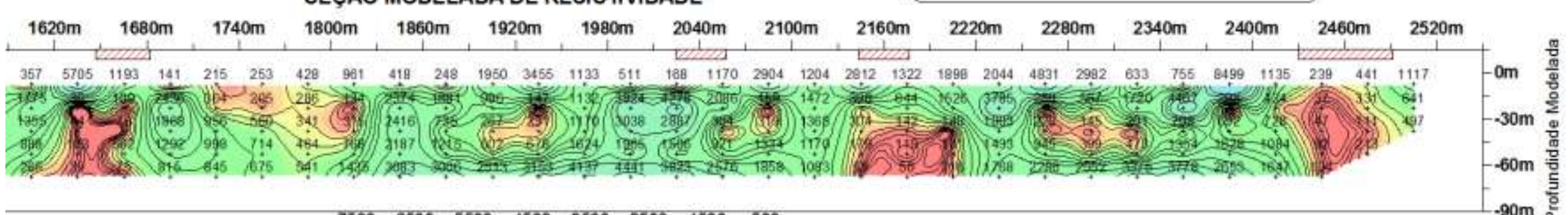

$\begin{array}{llllllll}7500 & 6500 & 5500 & 4500 & 3500 & 2500 & 1500 & 500\end{array}$

Figura 8 - Trecho A2 da Linha A

ESCALA CROMÁTICA DE RESISTIVIDADE (ohms $x$ metro)

Figura 8 - Trecho A2 da pseudo-seção e seção modelada de resistividade da Linha A

Figure 8 - Part A2 of pseudo-section and modeled section of resistivity of Line A 
LEVANTAMENTO DE ELETRORRESISTIVIDADE, ARRANJO DIPOLO-DIPOLO - LINHA B - $A B=M N=30 \mathrm{~m}$ PSEUDO-SEÇÃO DE RESISTIVIDADE APARENTE

SONDAGEM

P4-L3 (IMPRODUTIVA)
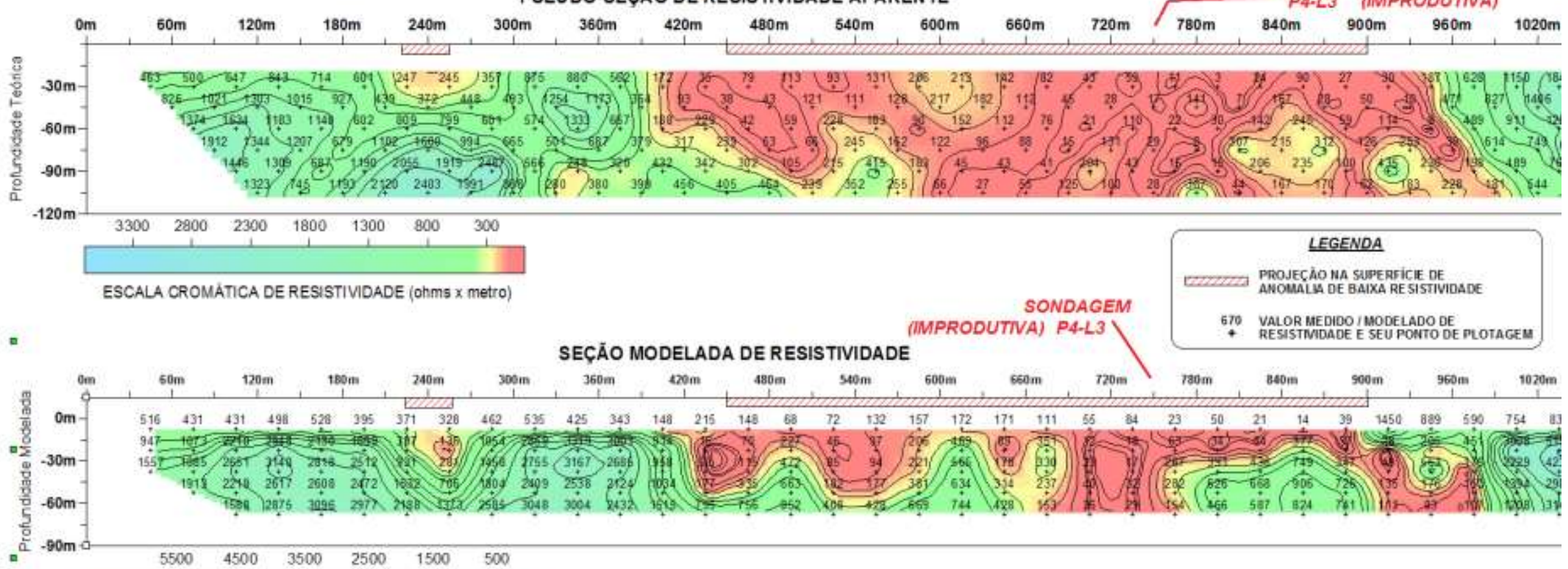

\begin{tabular}{|cccccc|}
5500 & 4500 & 3500 & 2500 & 1500 & 500 \\
\hline & 1 & 1 & 1 & 1 & 1 \\
\hline
\end{tabular}

ESCALA CROMÁTICA DE RESISTIVIDADE (ohms $x$ metro)

Figura 9 - Trecho B1 da Linha B

Figura 9 - Trecho B1 da pseudo-seção e seção modelada de resistividade da Linha B

Figure 9 - Part B1 of pseudo-section and modeled section of resistivity of Line B 


\section{LEVANTAMENTO DE ELETRORRESISTIVIDADE, ARRANJO DIPOLO-DIPOLO - LINHA B - $A B=M N=30 \mathrm{~m}$ SONDAGEM SONDAGEM}

P2-L7 (>75m3/h)

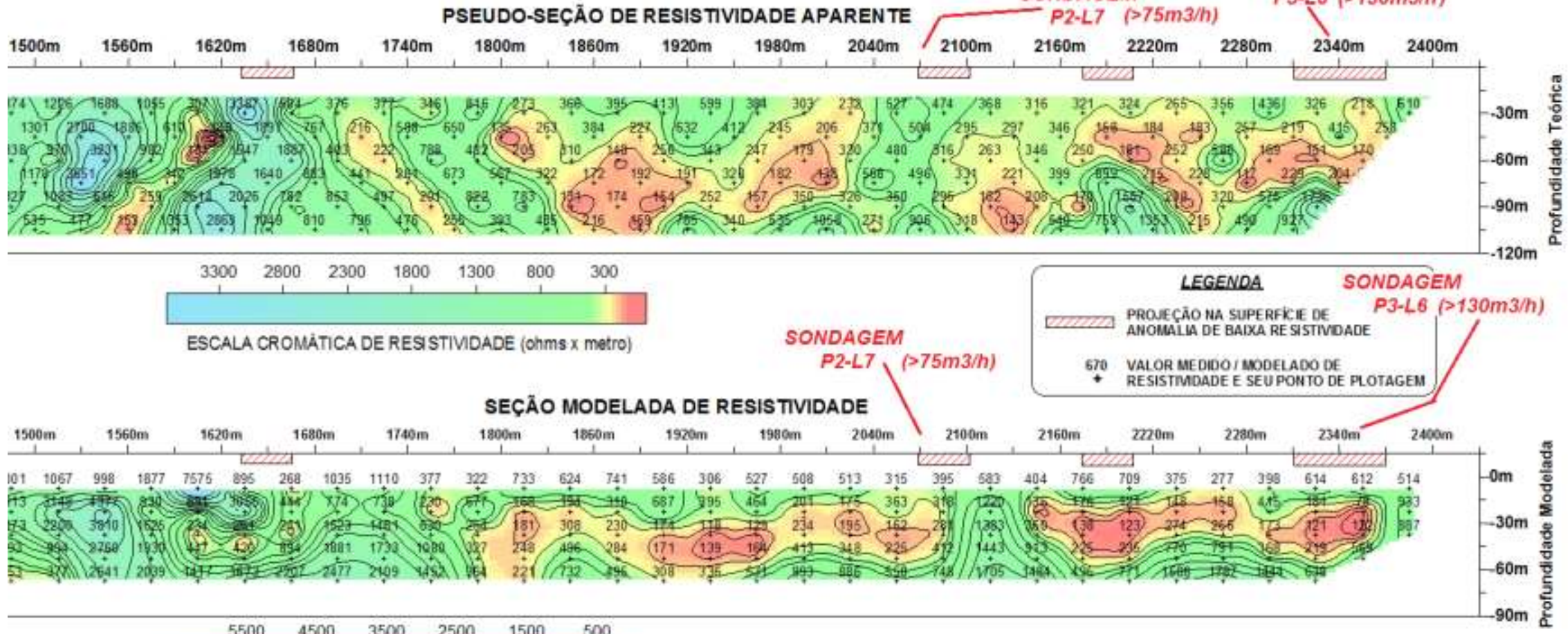

\begin{tabular}{ccccccc}
5500 & 4500 & 3500 & 2500 & 1500 & 500 \\
\hline 1 & 1 & 1 & 1 & 1 & 1 \\
\hline
\end{tabular}

\section{Figura 10 - Trecho B2 da Linha B}

Figura 10 - Trecho B2 da pseudo-seção e seção modelada de resistividade da Linha B

Figure 10 - Part B2 of pseudo-section and modeled section of resistivity of Line B 
LEVANTAMENTO DE ELETRORRESISTIVDADE, ARRANJO DIPOLO-DIPOLO - LINHA C - AB $=M N=30 \mathrm{~m}$
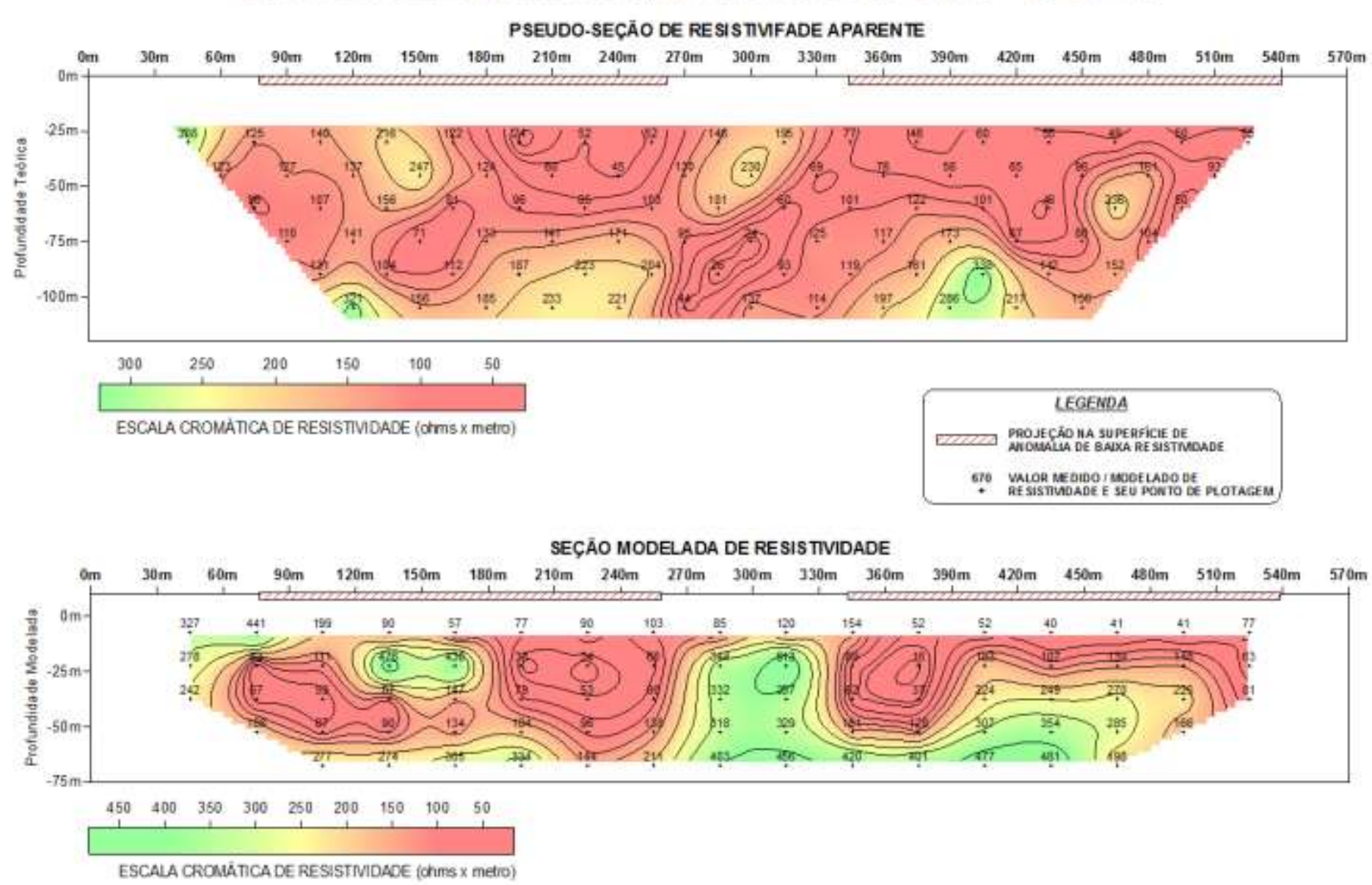

Figura 11 - Totalidade da pseudo-seção e seção modelada de resistividade da Linha C

Figure 11 - Pseudo-section and modeled resistivity section of line C 


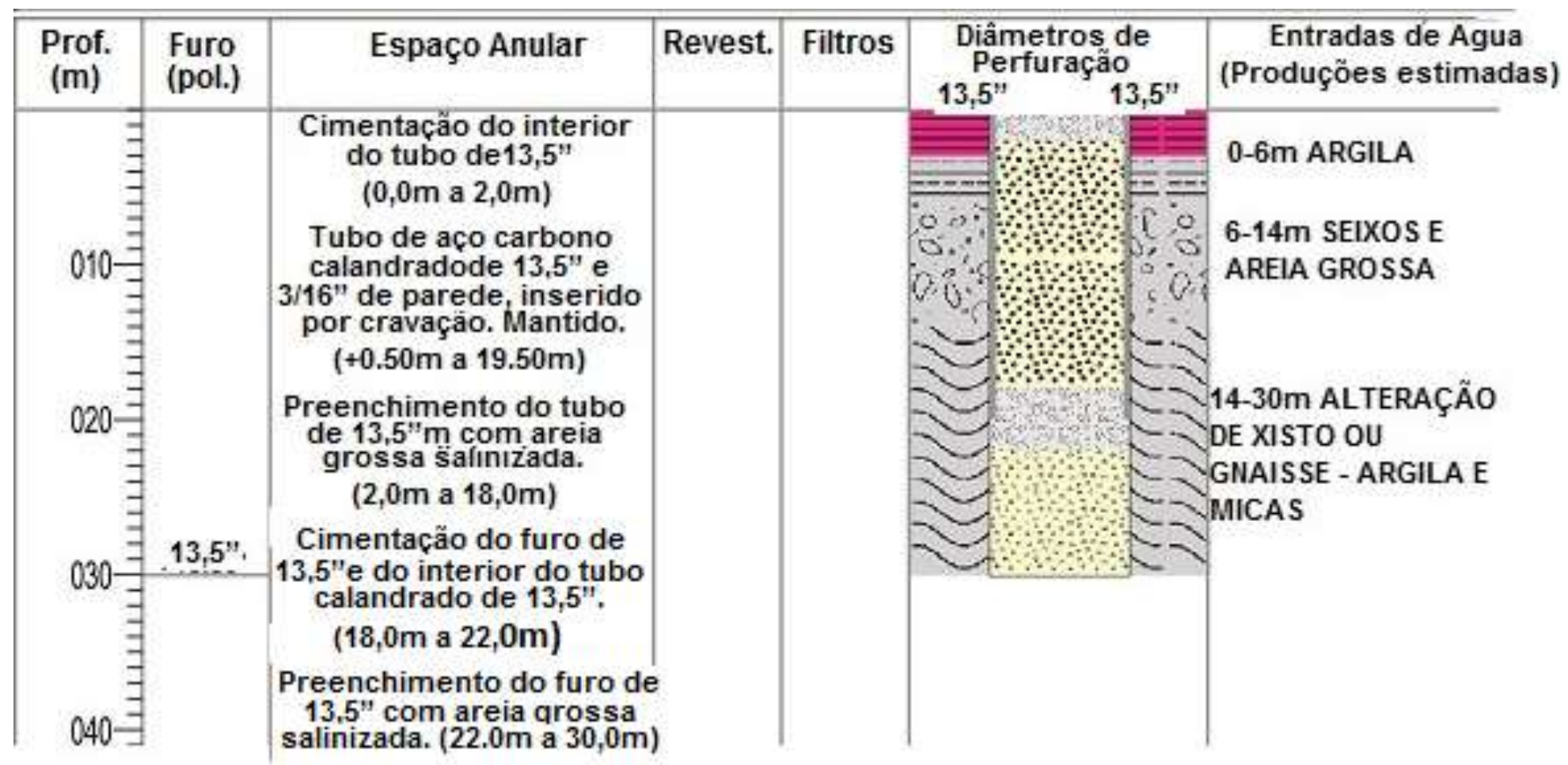

Figura 12 - Perfil do poço P4-L3, perfurado no ponto 750m da Linha B

Figure 12 - Well profile P4-L3, drilled in point 750 meters of Line B 


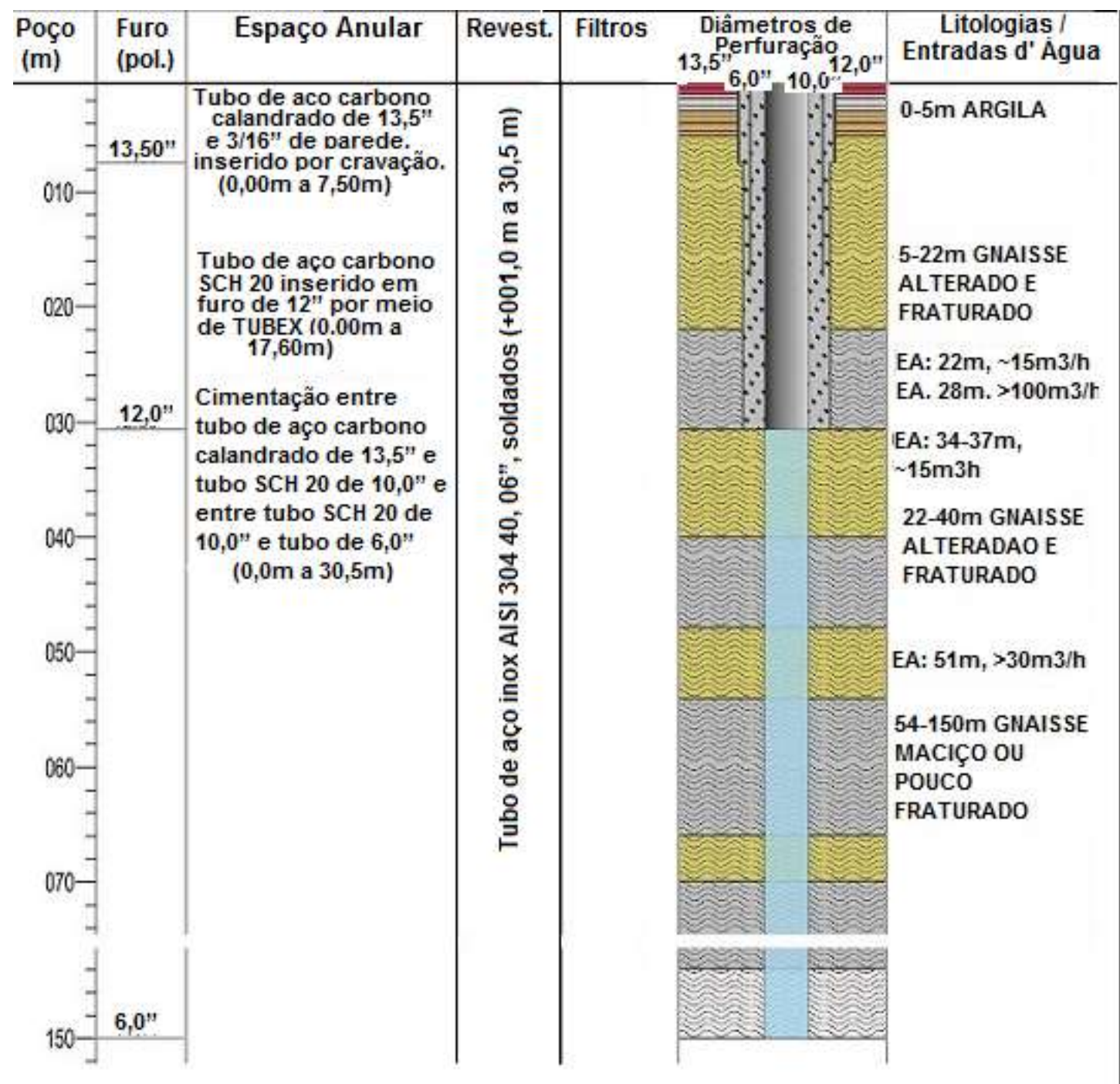

Figura 13 - Perfil do poço P2-L7, perfurado no ponto 2070m da Linha B Figure 13 - Well profile P2-L7, drilled in point 2070 meters of Line B 


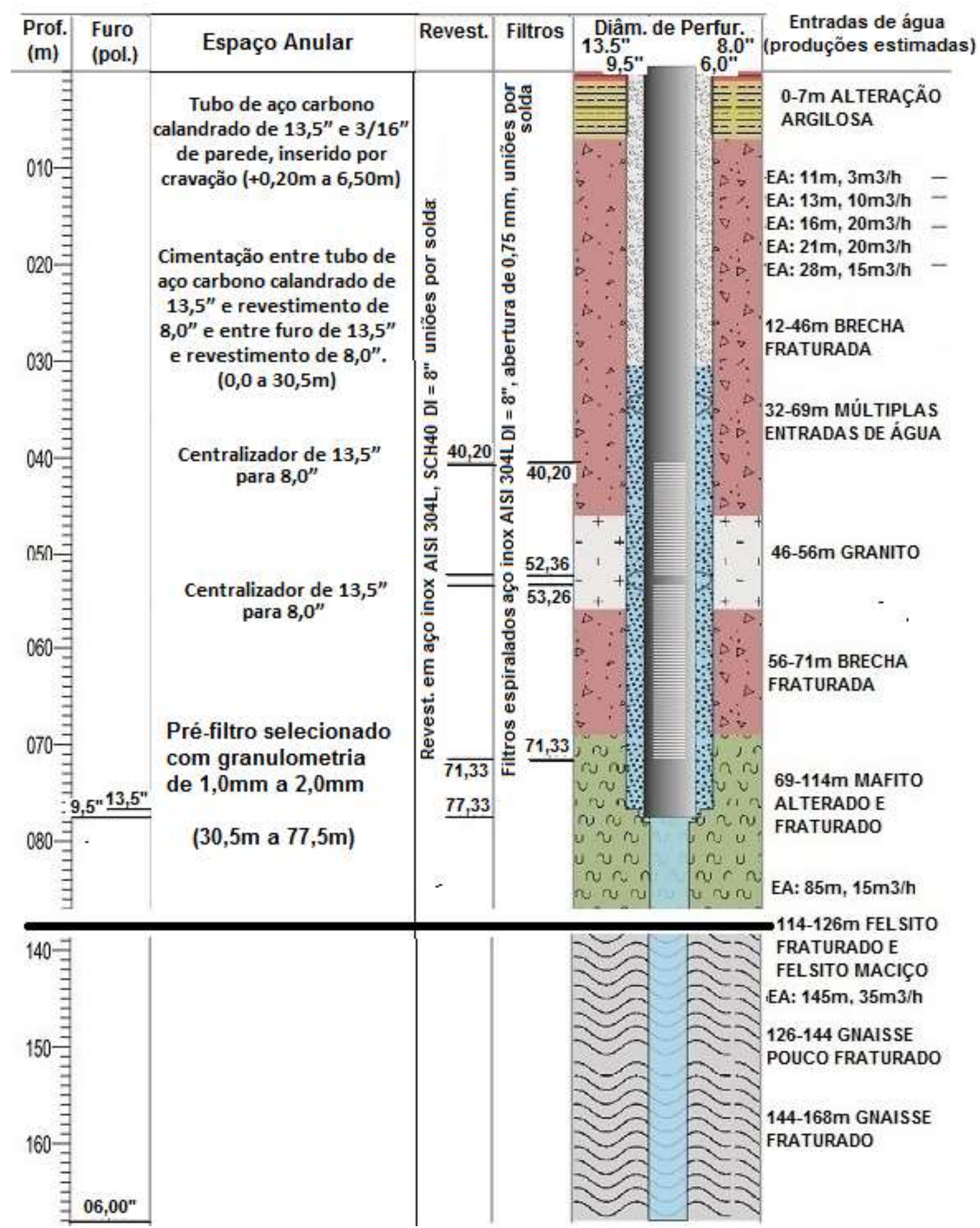

Figura 14 - Perfil do poço P3-L6, perfurado no ponto 2340m da Linha B

Figure 14 - Well profile P3-L6, drilled in point 2340 meters of Line B 


\section{PROCESSAMENTO DOS DADOS}

Os resultados obtidos com os ensaios de eletrorresistividade do caminhamento elétrico, dispositivo eletródico dipolo-dipolo, estão sendo apresentados nas figuras 7 a 11 sob a forma de pseudo-seções de resistividade aparente e como seções modeladas de resistividade.

As medidas de resistividade normalmente são apresentadas e interpoladas (contornos de isovalores) sob a forma de pseudoseções que dão uma representação visual do comportamento das resistividades em subsuperfície. No entanto, as formas dos contornos não dependem exclusivamente das distribuições de resistividades medidas, mas também da geometria da configuração de eletrodos empregada. Mesmo corpos de formas geométricas simples (retangulares, p. ex.), apresentam pseudo-seções completamente diferentes, conforme o arranjo de eletrodos empregado.

Nesse sentido, para obter-se uma seção de resistividade mais precisa, torna-se necessária a aplicação de processos de inversão nos dados que, em tese, conduz a uma aproximação razoável de modelos para diversas estruturas geológicas.

Estes processos de inversão foram empregados no tratamento dos dados do arranjo dipolo-dipolo deste levantamento, usando-se o já citado software RES2DINV (2001) que baseia-se no algoritmo desenvolvido por Loke \& Barker (1996a, 1996b) e faz a inversão dos dados por mínimos quadrados com vínculo de suavidade ("smoothness constrained least-square method"). As profundidades de investigação (default do software) fundamentam-se nas propostas por Edwards (1977) e são aproximadamente a metade daquela estimada por Hallof (1957), mas que podem ser modificadas pelo usuário do software.

Assim, as seções modeladas são resultantes de um processo automático de inversão 2-D que buscam estabelecer um modelo que se ajusta a uma possível distribuição real dos valores de resistividade em subsuperfície. As eventuais distorções nas pseudo-seções inerentes aos arranjos de eletrodos são, teorica- mente, eliminadas pelos processos de inversão.

Madrucci et al. (2005) empregando métodos geoelétricos (sondagens elétricas verticais e caminhamentos elétricos) em área selecionada a partir de fotografias aéreas definiram favorabilidades - detecção de fraturamentos - em área cristalina.

Pereira (2009) prospectou aquíferos fissurais empregando a eletrorresistividade associada à emanação natural de radônio, tendo obtido bons resultados com ambos os métodos. Verificou que, no caso de rochas graníticas (ácidas e ígneas e/ou metamórficas) existe uma estreita correlação entre fraturas, emanação de Rn e, consequentemente, favorabilidade à presença de aquíferos.

Também foi objeto da detecção de fraturas em rochas graníticas a dissertação de mestrado de Silva (2014), mas com a finalidade de selecionar rochas íntegras (sem fraturas) para extração de blocos graníticos de grande porte.

Gallas \& Verma (2006) abordam as profundidades de investigação, entre outras variáveis (mergulho, teores de material polarizável (Polarização Induzida - IP), espessuras etc), empregando modelos físicos cilíndricos e tabulares para modelagem analógica de resistividade (e IP) em laboratório. Gallas (2003) obteve resultados que demonstram a eficácia da eletrorresistividade e sugere aberturas mínimas de dipolo com base na expectativa da profundidade a que se encontram os alvos da prospecção - zonas falhadas/fraturadas sob cobertura de alteração em área de ocorrência de rochas cristalinas em São José do Rio Pardo/SP. Este informe foi determinante na escolha da abertura entre eletrodos empregada nesta pesquisa $(\mathrm{AB}=\mathrm{MN}=30 \mathrm{~m})$ e o número de níveis de investigação (06).

\section{INTERPRETAÇÃO E RESULTADOS ATINGIDOS}

A área é de ocorrência de rochas ígneas/metamórficas, principalmente de natureza granito-gnássicas e pode-se associar as zonas de resistividades mais baixas (cores 
amarelo-avermelhadas nas figuras), dependendo da forma assumida nas pseudo-seções e seções modeladas de resistividade, como locais associados a prováveis fraturamentos e/ou locais onde há aprofundamento do topo da rocha sã.

Neste contexto geológico, a presença de aquíferos ocorre, principalmente, em locais fraturados, uma vez que, contrariamente às rochas sedimentares, este tipo de litologia praticamente não apresenta porosidades primárias capazes de armazenar água. Por outro lado, conforme já citado, espessamentos de coberturas e/ou depósitos de tálus também podem ser favoráveis ao armazenamento de água explotável.

As fraturas e os citados espessamentos estão relacionados às baixas resistividades, devido ao fato de que a presença de fraturas, espessamentos do pacote solos+rocha alterada ou depósitos de tálus, usualmente estão preenchidos com água, o que enriquece o meio em íons em solução e partículas em suspensão. Como a condução da corrente elétrica em condições como as da natureza dá-se principalmente de forma iônica, ocorre diminuição das resistividades nestes locais.

Desta forma, os substratos com um eletrólito rico em íons e material particulado permitirão que a corrente elétrica se propague com maior facilidade, resultando em anomalias de baixas resistividades. Assim, é possível estabelecer-se uma correlação entre as zonas de resistividades mais baixas e eventuais falhamentos/fraturamentos (Gallas, 2000, 2003).

As baixas resistividades também indicam, ainda nestas áreas cristalinas, eventuais espessamentos de coberturas ou depósitos de tálus, definidos como depósitos de sopé de escarpas com fortes declividades originados principalmente por efeito da gravidade sobre fragmentos rochosos soltos. Tais feições também podem se constituir em aquíferos quando têm boas porosidades e permeabilidades.

Neste contexto geológico, as estruturas consideradas e detectadas pelos contrastes nas resistividades medidas, na maioria das vezes, podem estar associadas à presença de aquíferos subterrâneos.

Os resultados dos ensaios de eletrorresistividade permitiram estabelecer os locais de maiores e menores resistividades e estabelecer as seguintes correlações:

- Áreas menos resistivas (cores amarelo-avermelhadas nas figuras) correlacionam-se com zonas fraturadas/falhadas e possível depósito de tálus ou rochas xistosas e seus produtos de alteração.

- Áreas mais resistivas (cores verdeazuladas nas figuras) - correlação com possíveis rochas graníticas/gnássicas e locais pouco ou nada fraturados.

Diante do exposto e dos resultados apresentados, foram indicados os seguintes locais para locação de sondagens e futura instalação de poços para explotação dos aquíferos subterrâneos:

Zona interpretada como depósito de tálus ou rochas xistosas:

Entre pontos $660 \mathrm{~m}$ e $780 \mathrm{~m}$ da linha B.

Entre $720 \mathrm{~m}$ e $800 \mathrm{~m}$ da linha A.

Entre $210 \mathrm{~m}$ e $240 \mathrm{~m}$; entre $360 \mathrm{~m}$ e 390m da linha C.

\section{Fraturas/falhamentos:}

Linha A: ponto $90 \mathrm{~m}$, ponto $1.680 \mathrm{~m}$, ponto $2.040 \mathrm{~m}$, ponto $2.160 \mathrm{~m}$, entre $2.400 \mathrm{e}$ $2.460 \mathrm{~m}$.

Linha B: ponto $240 \mathrm{~m}$, ponto $1.650 \mathrm{~m}$, ponto $2.040 / 2.070 \mathrm{~m}$, ponto $2.190 \mathrm{~m}$, entre 2.310 e $2.370 \mathrm{~m}$.

Todos os pontos foram georreferenciados em coordenadas UTM

A Figura 5 contém uma Imagem Google Earth e localiza os levantamentos efetuados na área estudada devidamente georreferenciados.

As Figuras 7 a 11 trazem as pseudoseções de resistividade aparente e seções modeladas dos perfis. Ambas as formas de apresentação dos dados mostram contrastes de resistividade que podem ser correlacionados a aspectos litológicos em subsuperfície.

Conforme visto nestas figuras, são assinaladas nas mesmas, através de barras hachuradas vermelhas, as projeções na superfície de anomalias de resistividade que foram 
interpretadas como zonas de falhas/fraturamentos bem como uma zona extensa de resistividades bai-

xas.

A Figura 6 - mapa de resistividade do nível 4 e síntese dos ensaios geofísicos - visa representar em planta a distribuição das resistividades. Esta figura posiciona na área objeto os três perfis levantados e os pontos de medida em cada um deles. Também está delimitada nesta figura a faixa extensa de baixas resistividades.

Pelo observado na Figura 6, esta mostra que os alinhamentos detectados - constituídos pela correlação entre anomalias - têm direção principalmente SW-NE e situam-se na porção Norte da área. Somente um alinhamento, situado ao Sul, apresenta direção SE-NW.

A já citada porção de baixa resistividade foi identificada nas três linhas levantadas e tem aproximadamente $400 \mathrm{~m}$ de extensão na direção $\mathrm{N}-\mathrm{S}$, verificando-se desde a superfície até as últimas profundidades investigadas, conforme visto em todos os perfis e sua ocorrência é mostrada em planta na Figura 6.

A interpretação para este trecho extenso de resistividades baixas admite duas possibilidades: esta feição geológica/geofísica poderia estar relacionada a um possível depósito de tálus - um potencial aquífero - ou corresponder à presença de rochas xistosas e seus produtos de alteração, praticamente sem possibilidades de produzir água.

Infelizmente, em perfuração efetuada, confirmou-se a presença da alteração de xistos, confirmando-se o estrato hidrogeologicamente improdutivo.

O local desta sondagem negativa situa-se nas proximidades do ponto $750 \mathrm{~m}$ da linha B e foi denominado poço P4-L3 (Figura 9).

A descrição da perfuração é apresentada na Figura 12, que atingiu $30 \mathrm{~m}$ e, uma vez confirmada a alteração de xistos, foi suspensa nesta profundidade.

Por outro lado, as duas perfurações seguintes realizadas em outras anomalias de resistividades baixas resultaram em dois poços com excepcionais produções de águas de excelente qualidade, classificadas como minerais.

A primeira sondagem foi executada na linha B no ponto $2070 \mathrm{~m}$ e foi denominada de poço P2-L7 (Figura 10).

A descrição desta perfuração, que atingiu $150 \mathrm{~m}$ de profundidade, encontra-se na Figura 13 e interceptou gnaisses que, quando fraturados, apresentaram ótimas entradas de água (identificadas como EA, na figura).

A produção deste poço, após os testes preliminares de bombeamento, foi estimada da ordem de $75 \mathrm{~m}^{3} / \mathrm{h}$.

A segunda perfuração produtiva foi levada a cabo à altura do ponto $2340 \mathrm{~m}$, também na linha B, chamada de poço P3-L6 (Figura 10).

A descrição desta sondagem, que alcançou 168m, é apresentada na Figura 14, atravessando brechas, mafitos e granito/gnaisses com várias entradas de água (EA).

Os testes de bombeamento deste poço estimaram uma produtividade da ordem de $130 \mathrm{~m}^{3} / \mathrm{h}$.

\section{CONCLUSÕES E RECOMENDA- ÇÕES}

De acordo com as características geológicas da área estudada - rochas granitognáissicas - a detecção de falhas/fraturamentos ou outras feições correlacionáveis a potenciais aquíferos em subsuperfície foi o objetivo desta prospecção geofísica.

Conforme os resultados obtidos nos levantamentos de eletrorresistividade efetuados na área pesquisada obteve-se sucesso na detecção das áreas/locais favoráveis à locação de poços tubulares para explotação de águas subterrâneas.

Segundo o mapa geológico da área (figura 4, na escala 1:25:000) as direções principais das estruturas são NE-SW. Isto foi confirmado pelo levantamento geofísico em sua porção Sul, onde foi detectada uma estrutura com esta direção. No entanto, graças a esta pesquisa, as principais estruturas identificadas, têm direção perpendicular NW-SE, 
que aparecem somente no mapa 1:500.000.

Assim, o levantamento contribuiu de forma inequívoca e fundamental para o conhecimento do sistema aquífero local, uma vez que, sem os resultados obtidos, seriam desconhecidas estas estruturas locais que, após perfuradas, resultaram em poços com ótimas produtividades. Desta forma, esta pesquisa certamente servirá de guia para futuros trabalhos similares que vierem a ser efetuados na região.

Nos locais indicados como anômalos, de resistividades mais baixas, foram efetuadas perfurações que resultaram em poços de

\section{REFERÊNCIAS}

EDWARDS, L. S. A modified pseudo-section for resistivity and induced-polarization. Geophysics, v.3, p.78-95, 1977.

GALLAS, J. D. F. Principais métodos geoelétricos e suas aplicações em prospecção mineral, hidrogeologia, geologia de engenharia e geologia ambiental. Rio Claro, 174p. Tese (Doutorado em Geociências e Meio Ambiente) - Instituto de Geociências e Ciências Exatas, Universidade Estadual Paulista, 2000.

GALLAS, J. D. F. Prospecção de água subterrânea em aquíferos cristalinos com o emprego de métodos indiretos. Revista do Instituto Geológico, v. 24, p. 43-51, 2003.

GALLAS, J. D. F. \& VERMA, O. P. Resistividade e Polarização Induzida (IP) - Modelagem Analógica. Revista Brasileira de Geofísica, v 24, n.1, p.25-35, 2006.

HALLOF, P. G. On the interpretation of resistivity and induced polarization measurements: Cambridge, MIT, Ph. D. thesis, 1957.

LOKE, M.H. \& BARKER, R.D.Rapid least-squares inversion of apparent resistivity pseudosections by a quasi-Newton method. Geophysical Prospecting, 44, 131-152, 1996. excepcional produtividade em se tratando de aquíferos fraturados $\left(75 \mathrm{~m}^{3} / \mathrm{h}\right.$ e $\left.130 \mathrm{~m}^{3} / \mathrm{h}\right)$.

A exceção foi a anomalia extensa associada a xistos e seus produtos de alteração, que não resultou em um poço produtivo. Mas, mesmo neste caso, a interpretação geofísica/geológica já previa esta possibilidade.

Finalizando, o método da eletrorresistividade revelou-se mais uma vez ser uma ferramenta de prospecção indireta muito eficaz na detecção de zonas fraturadas e/ou falhadas e como de otimização na indicação de locais favoráveis à locação de poços para explotação de águas subterrâneas.

LOKE, M.H. \& BARKER, R.D.Practical techniques for $3 \mathrm{D}$ resistivity surveys and data inversion. Geophysical Prospecting, 44, 499-523, 1996b.

MADRUCCI, V.; TAIOLI, F.; ARAUJO, C. C. 2. Análise integrada de dados de sensoriamento remoto, geologia e geofísica no estudo de aquífero fraturado, Lindóia - SP. Revista Brasileira de Geofísica, v.23, n.4, p. 437-451, 2005.

ORELLANA, E. Prospeccion geoeletrica en corriente continua. Madrid: Paraninfo, 523 p., 1972.

PEREIRA, C. M. A. Mapeamento de aqüíferos fraturados empregando métodos geoelétricos e emanação natural de radônio. Instituto de Geociências, Universidade de São Paulo, São Paulo, 63 p. Dissertação (Mestrado em Geologia), 2009.

RES2DINV, ver. 3.4 for Windows 95/98/Me/2000 /NT/XP. Rapid 2-D Resistivity \& IP inversion using de last-squares method. Geotomo software, 2001.

SILVA, D. D. Caracterização de Áreas Favoráveis à Extração de Blocos Graníticos de Grande Porte Com o Uso da Eletrorresistividade. Instituto de Geociências, Universidade de São Paulo, São Paulo, 92 p. Dissertação (Mestrado em Geologia), 2014. 